\title{
A molecular outflow revealing star formation activity in the vicinity of the HII region G034.8-0.7 and the SNR W44
}

\author{
S. Paron ${ }^{1}$, M. E. Ortega ${ }^{1}$, M. Rubio ${ }^{2}$, and G. Dubner ${ }^{1}$ \\ 1 Instituto de Astronomía y Física del Espacio (IAFE), CC 67, Suc. 28, 1428 Buenos Aires, Argentina \\ e-mail: sparon@iafe.uba.ar \\ 2 Departamento de Astronomía, Universidad de Chile, Casilla 36-D, Santiago, Chile \\ Received 4 November 2008 / Accepted 6 February 2009
}

ABSTRACT

\begin{abstract}
Aims. This work aims at investigating the molecular gas component in the vicinity of two young stellar object (YSO) candidates identified at the border of the HII region G034.8-0.7 that is evolving within a molecular cloud shocked by the SNR W44. The purpose is to explore signatures of star forming activity in this complex region.

Methods. We performed a near and mid infrared study towards the border of the HII region G034.8-0.7 and observed a 90" $\times 90^{\prime \prime}$ region near $18^{\mathrm{h}} 56^{\mathrm{m}} 48^{\mathrm{s}},+01^{\circ} 18^{\prime} 45^{\prime \prime}(\mathrm{J} 2000)$ using the Atacama Submillimeter Telescope Experiment (ASTE) in the ${ }^{12} \mathrm{CO} J=3-2,{ }^{13} \mathrm{CO}$ $J=3-2, \mathrm{HCO}^{+} J=4-3$ and CS $J=7-6$ lines with an angular resolution of $22^{\prime \prime}$.

Results. Based on the infrared study we propose that the source 2MASS $18564827+0118471$ (IR1 in this work) is a YSO candidate. We discovered a bipolar ${ }^{12} \mathrm{CO}$ outflow in the direction of the line of sight and a $\mathrm{HCO}^{+}$clump towards IR1, confirming that it is a YSO. From the detection of the CS $J=7-6$ line we infer the presence of high density $\left(>10^{7} \mathrm{~cm}^{-3}\right)$ and warm $(>60 \mathrm{~K})$ gas towards IR1, probably belonging to the protostellar envelope where the YSO is forming. We investigated the possible connection of IR1 with the SNR and the HII region. By comparing the dynamical time of the outflows and the age of the SNR W44, we conclude that the possibility that the SNR triggered the formation of IR1 is unlikely. On the other hand, we suggest that the expansion of the HII region G034.8-0.7 is responsible for the formation of IR1 through the "collect and collapse" process.
\end{abstract}

Key words. ISM: molecules - ISM: clouds - ISM: jets and outflows - stars: formation

\section{Introduction}

The formation of stars occurs deep inside molecular clouds. In the standard scenario for the formation of an isolated low-mass star, a cold core contracts as magnetic and turbulent support are lost and subsequently collapses to form a protostar with a surrounding disk. Then, a stellar wind breaks out along the rotational axis of the system and drives a bipolar outflow, which gradually disperses the protostellar envelope revealing a premain sequence star with a disk (van Dishoeck \& Jørgensen 2008).

Several authors have studied the role of supersonic turbulence in star forming processes (e.g. Mac Low \& Klessen 2004). They suggest that when stars form the process is quick and dynamic, with gravitational collapse occurring at a rate controlled by supersonic turbulence. The balance between turbulence and gravity provides a natural explanation for the widely varying star formation rates seen on both cloud and galactic scales.

As the largest contribution to interstellar turbulence comes from supernova explosions, it is thought that the dominant driving mechanism in star-forming regions of galaxies, appears to be supernovae, while elsewhere coupling of rotation to the gas through magnetic fields or gravity may be important (Mac Low \& Klessen 2004).

Since the early suggestion made by Opik (1953), several numerical studies (e.g. Vanhala \& Cameron 1998; Melioli et al. 2006) proposed that supernova remnants (SNRs) can initiate the formation of new generations of stars. However, observational evidence of star formation triggered by SNRs is still scarce. The bright SNR W44 and the environment in which it is evolving is a good target to explore this possibility. W44 is one of the few demonstrated cases of an SNR-molecular cloud interacting system. Seta et al. (1998) discovered six giant molecular clouds that appear to be partially surrounding the remnant. Later, Seta et al. (2004) proved that some of these clouds (at $v_{\text {LSR }} \sim 45 \mathrm{~km} \mathrm{~s}^{-1}$ ) are physically interacting with the remnant on its southeastern side. The CO spectra corresponding to these molecular structures present wing emission that, as the authors conclude, unambiguously confirm the interaction between W44 and the molecular gas. The physical interaction of the blast wave with a clumpy interstellar medium is also supported by the detection of bright $\mathrm{OH}(1720 \mathrm{MHz})$ masers at LSR velocities approximately between 43 and $47 \mathrm{~km} \mathrm{~s}^{-1}$ (Hoffman et al. 2005, and references therein) and shocked $\mathrm{H}_{2}$ emission observed with Spitzer-IRAC (Reach et al. 2006). Many infrared sources are embedded in the portion of the giant molecular cloud that is being shocked by the SNR. In particular IRAS $18542+0114$, an IRAS point source that coincides with the border of the HII region G034.8-0.7 (Kuchar \& Clark 1997; Ortega et al. 2007) appears immersed in a region of shocked molecular gas interior to the SNR shell as seen in projection. Figure 1 shows the molecular cloud in the ${ }^{13} \mathrm{CO} J=1-0$ line (as extracted from the $\mathrm{GRS}^{1}$ ) from the average between 40 and $50 \mathrm{~km} \mathrm{~s}^{-1}$, the velocity range where the molecular cloud peaks, and also in coincidence with the systemic velocity of W44. The radio continuum of the SNR and the HII region at $20 \mathrm{~cm}$ as extracted from the MAGPIS (Helfand et al. 2006) are depicted in thick blue contours.

The distance to W44 and the shocked molecular cloud was estimated to be $\sim 3 \mathrm{kpc}$ (Radhakrishnan et al. 1972; Caswell et al. 1975; Seta et al. 2004). Taking into account the recombination

1 Galactic Ring Survey (Jackson et al. 2006). 


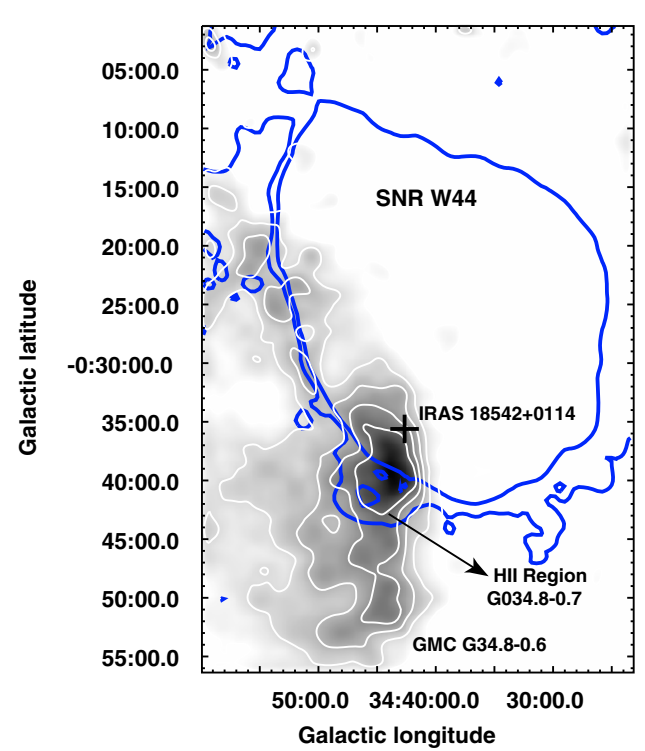

Fig. 1. ${ }^{13} \mathrm{CO} J=1-0$ line averaged between 40 and $50 \mathrm{~km} \mathrm{~s}^{-1}$ showing the giant molecular cloud GMC G34.8-0.6. The thick blue contours outline the radio continuum emission of the SNR W44 and the HII region G034.8-0.7 at $20 \mathrm{~cm}$. The cross shows the position of IRAS $18542+0114$.

line velocity of $52.1 \pm 3.6 \mathrm{~km} \mathrm{~s}^{-1}$ derived by Lockman (1989) and the HI absorption study performed by Ortega et al. (2007), the same distance as the SNR is suggested for the HII region G034.8-0.7. In what follows we adopt $3 \mathrm{kpc}$ as the distance for the SNR W44, the giant molecular cloud GMC G34.8-0.6 and the HII region G034.8-0.7.

IRAS 18542+0114 (cross in Fig. 1) is resolved into several sources in the Two Micron All-Sky Point Source Catalog (2MASS, Cutri et al. 2003), and according to color criteria (see Sect. 3.1) some of them are young stellar object (YSO) candidates.

Thus, the scenario is that there are YSO candidates within a shocked molecular cloud located in a region peripheric to a SNR and simultaneously on the border of an expanding HII region.

In this work we present a near and mid infrared study and molecular observations in the ${ }^{12} \mathrm{CO}$ and ${ }^{13} \mathrm{CO} J=3-2$, $\mathrm{HCO}^{+} J=4-3$ and CS $J=7-6$ lines towards IRAS $18542+0114$ carried out with the purpose of exploring the star forming activity in the region and its connection with the local SNR and the HII region.

\section{Observations}

The molecular observations were performed on June 25, 2008 with the $10 \mathrm{~m}$ Atacama Submillimeter Telescope Experiment (ASTE, Ezawa et al. 2004). We used the CATS345 GHz band receiver, which is a two-single band SIS receiver remotely tunable in the LO frequency range of 324-372 GHz. We simultaneously observed ${ }^{12} \mathrm{CO} J=3-2$ at $345.796 \mathrm{GHz}$ and $\mathrm{HCO}^{+} J=$ $4-3$ at $356.734 \mathrm{GHz}$, mapping a region of $90^{\prime \prime} \times 90^{\prime \prime}$ centered at the position of IRAS $18542+0114\left(\mathrm{RA}=18^{\mathrm{h}} 56^{\mathrm{m}} 47.8^{\mathrm{s}}\right.$, dec. $=+01^{\circ} 18^{\prime} 45^{\prime \prime}$, J2000). The mapping grid spacing was $10^{\prime \prime}$ and the integration time was $72 \mathrm{~s}$. per pointing. Additionally we observed ${ }^{13} \mathrm{CO} J=3-2$ at $330.588 \mathrm{GHz}$ and $\mathrm{CS} J=7-6$ at $342.883 \mathrm{GHz}$ towards the centre of the region. All the observations were performed in position switching mode. The off

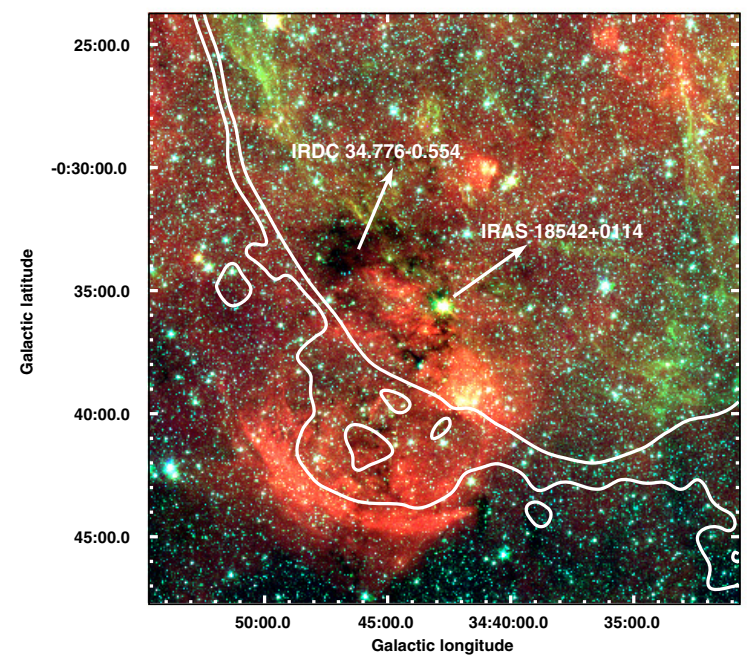

Fig. 2. Spitzer-IRAC three color image $(3.5 \mu \mathrm{m}=$ blue, $4.5 \mu \mathrm{m}=$ green and $8 \mu \mathrm{m}=$ red) of a region about $22^{\prime} \times 22^{\prime}$ around IRAS $18542+0114$. The contours correspond to the continuum emission at $20 \mathrm{~cm}$ of the SNR W44 and the HII region G034.8-0.7.

position was $\mathrm{RA}=18^{\mathrm{h}} 55^{\mathrm{m}} 30^{\mathrm{s}}$, dec. $=+01^{\circ} 39^{\prime} 55^{\prime \prime}(\mathrm{J} 2000)$ that was checked to be free of emission.

We used the XF digital spectrometer with a bandwidth and spectral resolution set to $128 \mathrm{MHz}$ and $125 \mathrm{kHz}$, respectively. The velocity resolution was $0.11 \mathrm{~km} \mathrm{~s}^{-1}$ and the half-power beamwidth (HPBW) was $22^{\prime \prime}$ at $345 \mathrm{GHz}$. The system temperature varied from $T_{\text {sys }}=400$ to $700 \mathrm{~K}$. The typical rms noise (in units of $T_{\mathrm{mb}}$ ) ranged between 0.1 and $0.4 \mathrm{~K}$ and the main beam efficiency was $\eta_{\mathrm{mb}} \sim 0.65$.

The spectra were Hanning smoothed to improve the signalto-noise ratio and only linear or/and some third order polynomia were used for baseline fitting. The spectra were processed using the XSpec software package developed at the Onsala Space Observatory.

\section{Results}

\subsection{Infrared emission}

Figure 2 shows a Spitzer-IRAC three color image of a region about $22^{\prime} \times 22^{\prime}$ around IRAS $18542+0114$ as extracted from GLIMPSE (Fazio et al. 2004; Werner et al. 2004). The three IR bands are $3.6 \mu \mathrm{m}$ (in blue), $4.5 \mu \mathrm{m}$ (in green) and $8 \mu \mathrm{m}$ (in red). Contours of the radio continuum emission at $20 \mathrm{~cm}$ of the SNR W44 and the HII region G034.8-0.7 are included.

The IRAC $4.5 \mu \mathrm{m}$ band contains both $\mathrm{H}_{2}(v=0-0, \mathrm{~S}(9$, $10,11))$ lines and $\mathrm{CO}(v=1-0)$ band heads. As noticed by Cyganowski et al. (2008), all of these lines may be excited by shocks, such as those expected when protostellar outflows crash into the ambient ISM. In Fig. 2 it can be noticed that IRAS $18542+0114$ appears slightly extended in the $4.5 \mu \mathrm{m}$ emission (green), therefore suggesting that it may be a YSO outflow candidate. It is important to note the difference between the emission from this YSO candidate and from the gas shocked by the SNR, both seen in green. The first one is bright and concentrated around IRAS $18542+0114$, while the second one is seen as diffuse filaments. From Fig. 2 the presence of an infrared dark cloud is also evident (IRDC 34.776-0.554 as catalogued in the Glimpse Dark Cloud Catalog). IRAS $18542+0114$ is seen in projection over a border of this infrared dark cloud (IRDC). The 


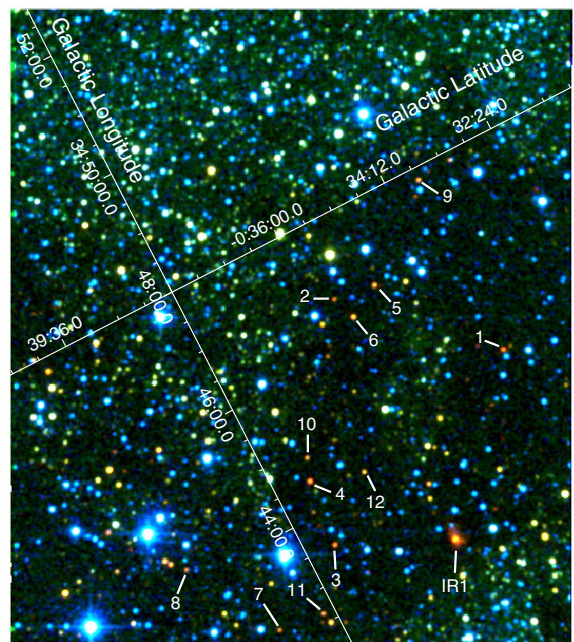

Fig. 3. 2MASS $J H K$ three-color image towards IRDC 34.776-0.554. The most reddened NIR sources are indicated with numbers. IRAS $18542+0114$ is resolved into several 2MASS sources. The brightest and the most reddened one is 2 MASS $18564827+0118471$.

IRDCs have been shown in recent years to be sites of the earliest stages of star formation (Rathborne et al. 2006, 2007).

Figure 3 shows near infrared (NIR) $J H K$ three-color image in a region of about $10^{\prime} \times 10^{\prime}$ that contains IRDC $34.776-0.554 \mathrm{ob}-$ tained from the 2MASS Survey (Skrutskie et al. 2006). The most reddened NIR sources are indicated with numbers. Among these sources is included the bright 2MASS $18564827+0118471$, the possible counterpart of IRAS $18542+0114$.

To look for primary tracers of star formation activity in the vicinity of the IRDC, we used the 2MASS All-Sky Point Source Catalogue in bands $J(1.25 \mu \mathrm{m}), H(1.65 \mu \mathrm{m})$ and $K(2.17 \mu \mathrm{m})$. We performed photometry in the region shown in Fig. 3. Figure 4 shows the $(H-K \mathrm{~s})$ versus $(J-H)$ color-color $(\mathrm{CC})$ diagram. We selected sources with $K \mathrm{~s} \leq 15$ and with detection in at least two bands. The typical color errors are about $5 \%$. Based on the above criteria we found 1029 sources in the mentioned region. Following Hanson et al. (1997), we calculated for each source the parameter $q=(J-H)-1.83 \times(H-K \mathrm{~s})$, which determines the distance to the reddened vector. YSO candidates, located at the Infrared Excess Sources region in Fig. 4, have $q$-values less than -0.15 . From this criterium we found 364 YSO candidates. The most reddened sources shown in Fig. 4 as circled dots present the best quality detection in the $H$ and $K$ bands. The numbers correspond to the numbered sources in Fig. 3. Their locations in the CC diagram indicate that they are likely YSOs, where the NIR excess around protostars is due to the optically thick circumstellar disk/envelopes. Figure 4 also shows two different groups of sources located between the parallel dashed lines. The first one, close to the origin of the diagram corresponds to several blue sources which can be seen in Fig. 3. They are probably foreground stars not related to the IRDC, and as can be noticed in the $\mathrm{CC}$ diagram they are clustered near the main sequence curve with visual extinction values from $A_{V} \sim 3$ to $8 \mathrm{mag}$. The second group is located between a visual extinction that ranges from $A_{V} \sim 18$ to $28 \mathrm{mag}$. Most of these sources correspond to background and possible molecular cloud embedded main sequence stars.

Figure 5 displays the spatial distribution of the most reddened sources mentioned above over the IRAC $8 \mu \mathrm{m}$ band emission. The emission in this IR band clearly depicts the border of the HII region, with the IR radiation mainly originating in the

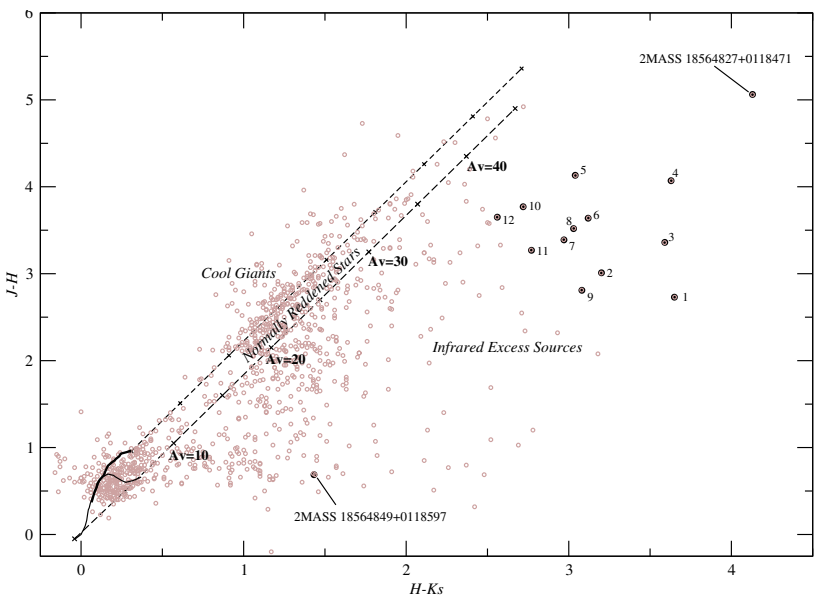

Fig. 4. Color-color diagram of the infrared sources in the vicinity of IRDC 34.776-0.554. The two solid curves represent the location of the main sequence (thin line) and the giant stars (thicker line) derived from Bessell \& Brett (1988). The parallel dashed lines are reddening vectors with the crosses placed at intervals corresponding to five magnitudes of visual extinction. We have assumed the interstellar reddening law of Rieke \& Lebofsky (1985) $\left(A_{J} / A_{V}=0.282 ; A_{H} / A_{V}=0.175\right.$ and $A_{K} / A_{V}=0.112$ ). The plot is classified into three regions: cool giants, normally reddened stars and infrared excess sources. The most reddened sources are indicated as circled dots and the numbers correspond to the numbered sources of Fig. 3.

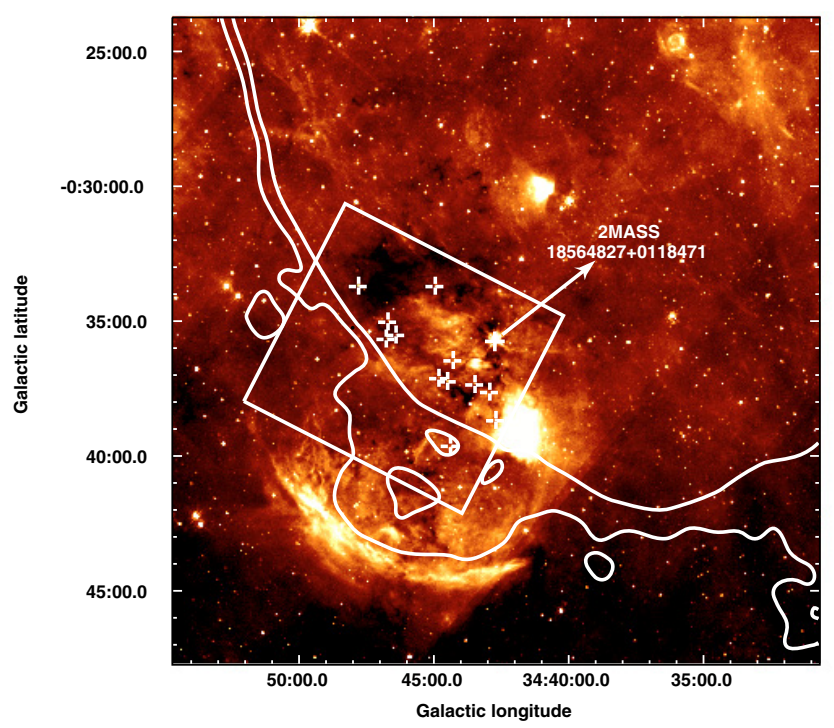

Fig. 5. IRAC $8 \mu \mathrm{m}$ band emission obtained from GLIMPSE. The crosses indicate the most reddened sources obtained from the photometric study performed in the area indicated by the box. The box represents the same region as shown in Fig. 3.

policyclic aromatic hydrocarbons (PAHs). The figure also includes the contours of the radio continuum emission of the SNR and the HII region and a box that indicates the region where the photometric study was performed. From this figure, it is seen that the most reddened sources lie on the border of the HII region that is seen in projection interior to the SNR shell. The location of these sources delineates the border of IRDC 34.776-0.554.

Figure 6 shows the IRAC color-color diagram for the most reddened sources highlighted and numbered in Fig. 3. The regions that indicate the stellar evolutionary stages are based on the criteria presented by Allen et al. (2004). The sources IR1 


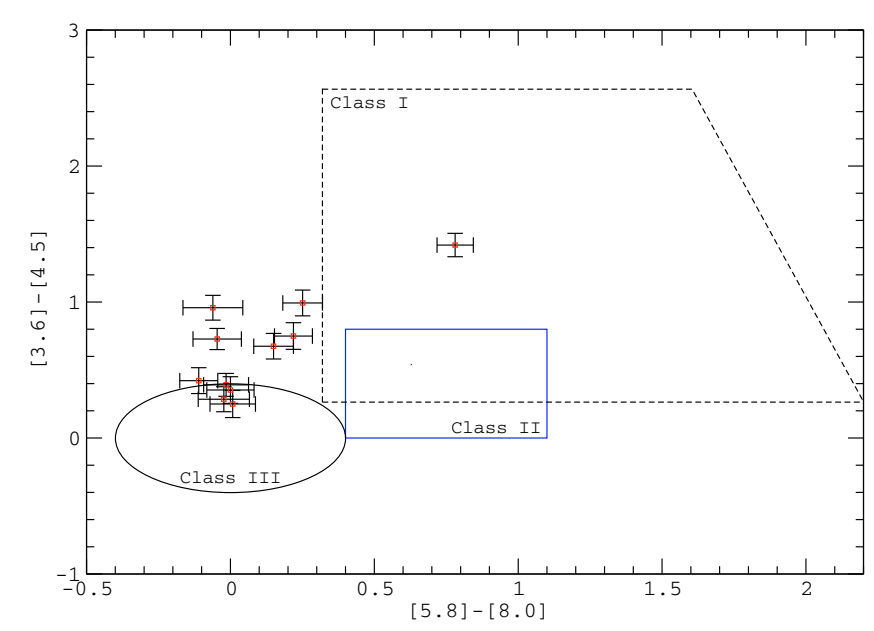

Fig. 6. GLIMPSE color-color diagram, [3.6]-[4.5] versus [5.8]-[8.0], for the most reddened sources from Fig. 3. Class I and Class II regions are indicated following Allen et al. (2004). The ellipse centered at 0, 0 encloses the region of main sequence and giant stars.

and 8 are not included because they are not detected in some IRAC bands. Only source 7 lies in the region of protostars with circumstellar envelopes (Class I), the sources 2, 5, 6, 10 and 12 lie in the region of the main sequence and giant stars (Class III). No source occupies the region of young stars with only disk emission (Class II). However the sources 1, 3, 4, 9 and 11 , located outside the delimited regions, could therefore be reddened Class II objets (Allen et al. 2004).

In particular, in a region of $90^{\prime \prime} \times 90^{\prime \prime}$ towards IRAS $18542+0114$ (IR1), the region analyzed in molecular lines (see next sections), the above 2MASS color criteria (Hanson et al. 1997) identifies two sources: 2MASS 18564827+0118471, the most reddened source in the whole studied region (box in Fig. 5), and 2MASS $18564849+0118597$, both indicated in the CC diagram (Fig. 4). In what follows we analyze the molecular gas in this region.

\subsection{Molecular emission}

\subsection{1. $\mathrm{HCO}^{+} J=4-3$}

Figure 7 shows a $90^{\prime \prime} \times 90^{\prime \prime}$ map obtained towards the infrared source IRAS $18542+0114$ in the $\mathrm{HCO}^{+} J=4-3$ line integrated between 40 and $50 \mathrm{~km} \mathrm{~s}^{-1}$. As noticed above, in this region there are two 2MASS sources, YSO candidates: 2MASS $18564827+0118471$ (source 1 in Fig. 7, hereafter IR1) and 2MASS 18564849+0118597 (source 2 in Fig. 7, hereafter IR2).

In Fig. 7 a dense $\mathrm{HCO}^{+}$clump is evident with IR1 exactly lying over the $\mathrm{HCO}^{+}$maximum and IR2 towards a border of the clump. By assuming as the limit of the $\mathrm{HCO}^{+} J=4-3$ structure the $3.5 \mathrm{~K} \mathrm{~km} \mathrm{~s}^{-1}$ contour ( $\sim 7$ times above the noise level), we can estimate a radius of $\sim 25^{\prime \prime}$ for this clump, $0.36 \mathrm{pc}$ at the adopted distance of $3 \mathrm{kpc}$.

Figure 8 shows an $\mathrm{HCO}^{+} J=4-3$ spectrum obtained from the average over the whole observed feature. From these observations we derived line parameters of the $\mathrm{HCO}^{+} J=4-3$ that are presented in Table 1 . The average main-beam brightness temperature $\left\langle T_{\mathrm{mb}}\right\rangle$, center line velocity, line width (FWHP), the average integrated $\mathrm{HCO}^{+}$intensity $\left\langle\int T_{\mathrm{mb}} \mathrm{d} v\right\rangle$ and the radius, are given in Cols. 1 to 5 , respectively. Errors are formal $1 \sigma$ value for the model of the Gaussian line shape.

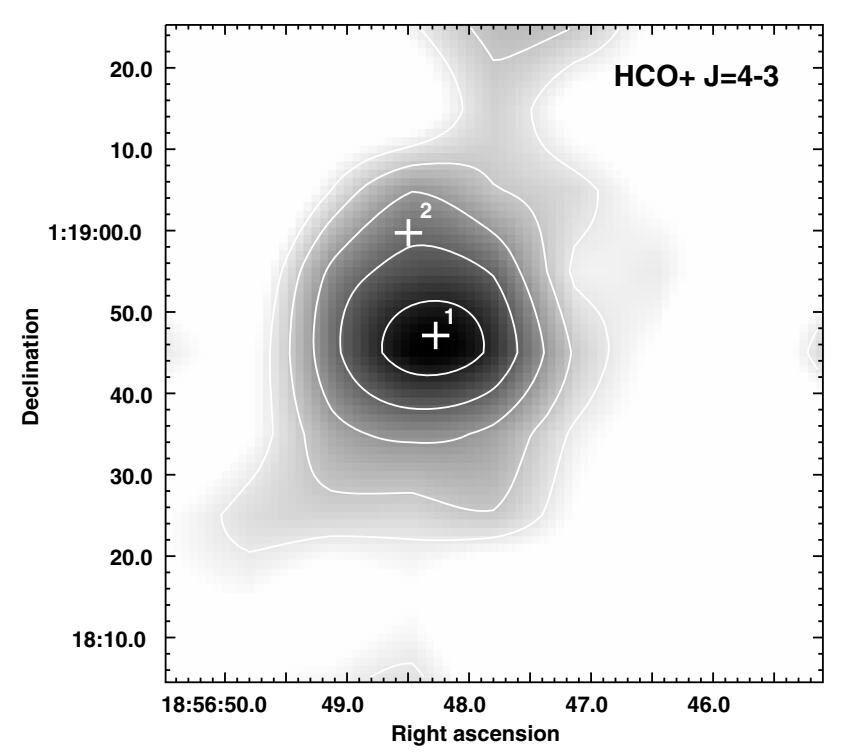

Fig. 7. $\mathrm{HCO}^{+} J=4-3$ emission integrated between 40 and $50 \mathrm{~km} \mathrm{~s}^{-1}$ towards the infrared source IRAS $18542+0114$. The contours levels are $3.5,5,7,9$ and $11 \mathrm{~K} \mathrm{~km} \mathrm{~s}^{-1}$. The crosses indicate the positions of the 2MASS point sources that according to the color criteria described in the text could be YSOs. The angular resolution is $\sim 22^{\prime \prime}$ and the $\sigma_{\mathrm{rms}} \sim 0.5 \mathrm{~K} \mathrm{~km} \mathrm{~s}^{-1}$.

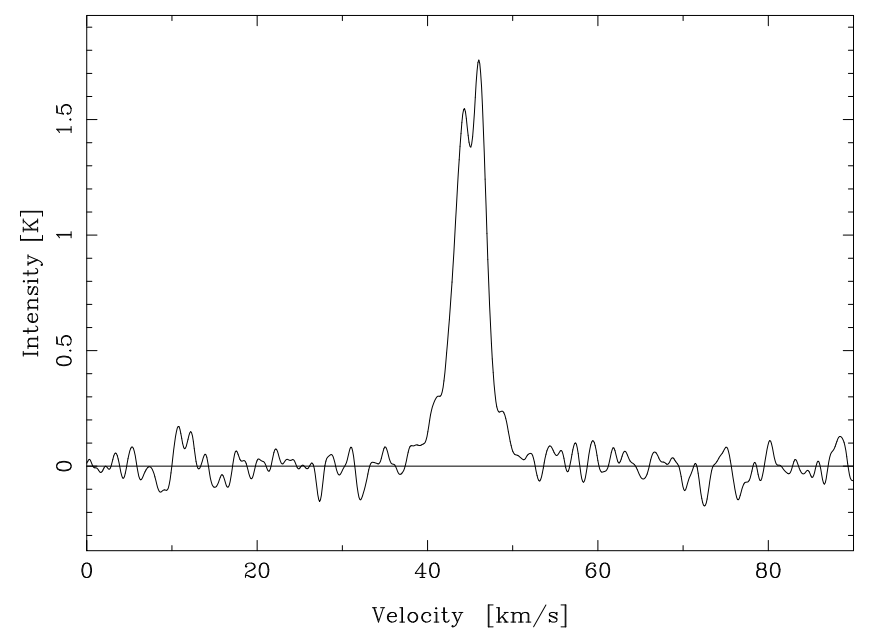

Fig. 8. $\mathrm{HCO}^{+} J=4-3$ average profile of the region where emission is detected. The rms noise is $\sigma_{\mathrm{rms}} \sim 0.03 \mathrm{~K}$.

The $\mathrm{HCO}^{+} J=4-3$ profile presented in Fig. 8 presents a dip at $v \sim 45 \mathrm{~km} \mathrm{~s}^{-1}$, the central velocity of the whole molecular complex. Only the profiles towards the center of the clump, that is, towards IR1, clearly display this feature (see Fig. 9). Such a dip can be interpreted as a self-absorption effect caused by less excited gas. As Hiramatsu et al. (2007) propose, this kind of spectral feature may indicate a significant population of the $J=3$ level in the outer gas, that requires a moderately high density but with lower $J=4-3$ excitation temperature than in the center of the clump. Such an effect might be revealing the existence of a density gradient in the clump.

We have also analyzed the existence of a possible blue asymmetry which would be evidence of infalling gas towards a protostar (Leung \& Brown 1977; Zhou 1992). In the star formation model of an infalling envelope, the blueshifted component is stronger than the redshifted one. This is due to the fact that the 
Table 1. Molecular clump parameters from the $\mathrm{HCO}^{+} J=4-3$ emission.

\begin{tabular}{ccccc}
\hline \hline $\begin{array}{c}\left\langle T_{\mathrm{mb}}\right\rangle \\
(\mathrm{K})\end{array}$ & $\begin{array}{c}V_{\mathrm{lsr}} \\
\left(\mathrm{km} \mathrm{s}^{-1}\right)\end{array}$ & $\begin{array}{c}\Delta v \\
\left(\mathrm{~km} \mathrm{~s}^{-1}\right)\end{array}$ & $\begin{array}{c}\left\langle T_{\mathrm{mb}} \mathrm{d} v\right\rangle \\
\left(\mathrm{K} \mathrm{km} \mathrm{s}^{-1}\right)\end{array}$ & $\begin{array}{c}R \\
(\mathrm{pc})\end{array}$ \\
\hline $1.65 \pm 0.20$ & $45.10 \pm 0.30$ & $4.40 \pm 0.60$ & $8.00 \pm 1.00$ & 0.36 \\
\hline
\end{tabular}

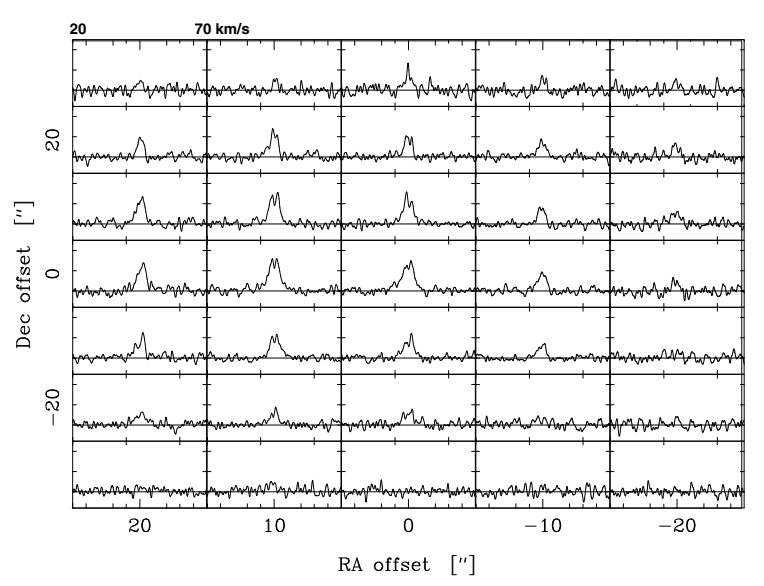

Fig. 9. $\mathrm{HCO}^{+} J=4-3$ spectra obtained towards IR1, whose offset position is approximately $\left(10^{\prime \prime}, 0^{\prime \prime}\right)$. The velocity range goes from 20 to $70 \mathrm{~km} \mathrm{~s}^{-1}$ and is shown at the top left profile. Note the profiles that present a dip at $v \sim 45 \mathrm{~km} \mathrm{~s}^{-1}$ near the center of the surveyed region.

redshifted component is absorbed by the cooler infalling gas in the near half of the envelope along the line of sight. We did not find such an asymmetry in our spectra. However, as discussed by Gregersen et al. (1997, 2000), the blue asymmetry line profiles do not appear in any of the known YSOs. One reason could be that if a redshifted outflow exists in the telescope beam, the redshifted components absorbed by the infalling envelope would be compensated.

\subsection{2. ${ }^{12} \mathrm{CO} J=3-2$}

Analyzing the ${ }^{12} \mathrm{CO} J=3-2$ data we find that the profiles towards IR1 are remarkably broader than those towards the border of the surveyed region. For comparison Fig. 10 shows two spectra: the upper one, obtained towards the center of the region and the bottom one towards the edge of the map. Such phenomena can be interpreted as evidence of an outflow observed along the line of sight driven by the source IR 1 . In effect, the blue component extends from 27 to $37 \mathrm{~km} \mathrm{~s}^{-1}$ while the red component goes from 50 to $63 \mathrm{~km} \mathrm{~s}^{-1}$. Figure 11 displays the ${ }^{12} \mathrm{CO} J=3-2$ emission integrated in both velocity ranges. The blue and red shifted components are presented in thin and thick contours, respectively. The crosses indicate the positions of the 2MASS sources IR1 and IR2.

The ${ }^{12} \mathrm{CO} J=3-2$ line almost always appears self-absorbed towards star-forming regions and often reveals information on the gas kinematics (Johnstone et al. 2003). Inspecting our data, most of the ${ }^{12} \mathrm{CO}$ profiles in the region have a dip at $v \sim$ $44 \mathrm{~km} \mathrm{~s}^{-1}$. This velocity is very close to the peak velocity of the ${ }^{13} \mathrm{CO} J=3-2$ line (see Sect. 3.2.3), which is an optically thinner line. Such correspondence strongly suggests that the dip in the ${ }^{12} \mathrm{CO}$ profiles is indeed caused by self-absorption by less excited gas (see e.g. Zhou et al. 1993). Thus we can discard the possibility of two ${ }^{12} \mathrm{CO}$ emission components with different velocities along the line of sight.

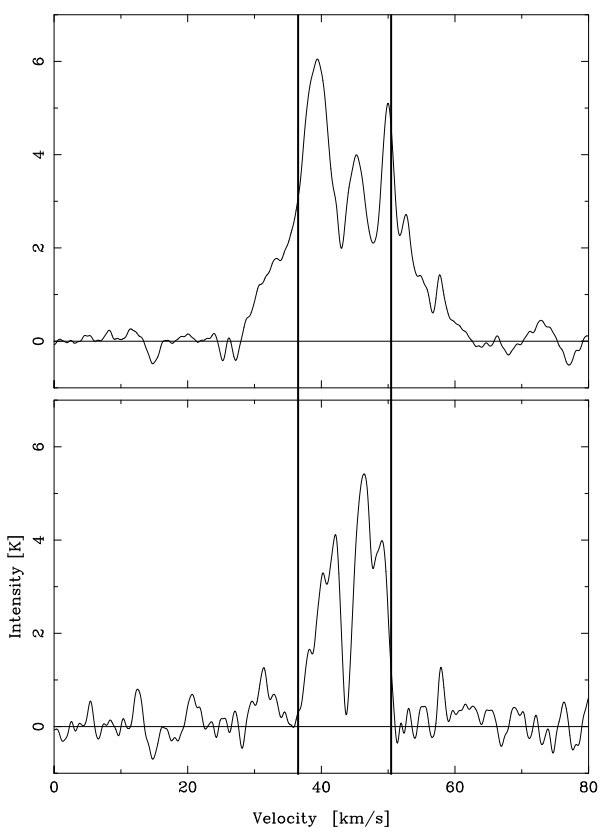

Fig. 10. ${ }^{12} \mathrm{CO} J=3-2$ spectra. Upper: spectrum towards the center of the observed region. Bottom: spectrum towards the edge of the region. The borders between outflow and line core are shown with two vertical lines. The rms noise is $\sigma_{\mathrm{rms}} \sim 0.15 \mathrm{~K}$.

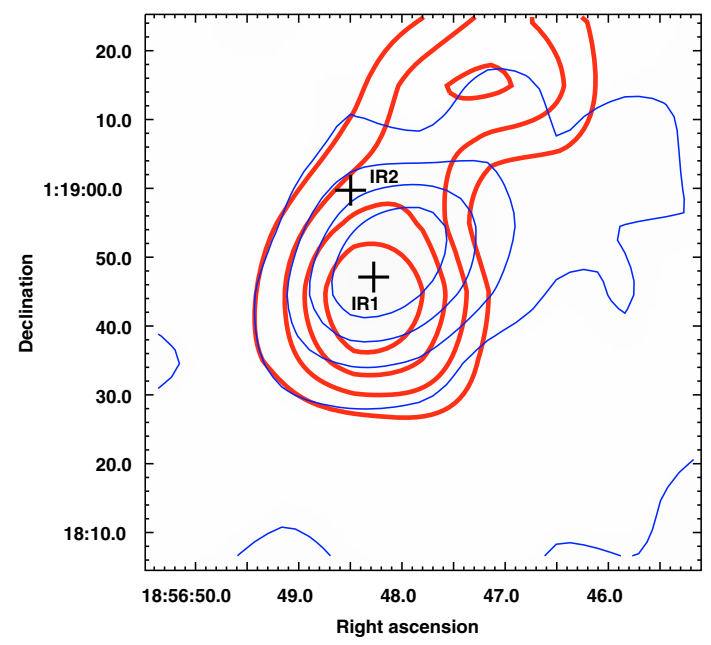

Fig. 11. The thin contours represents the ${ }^{12} \mathrm{CO} J=3-2$ emission integrated between 27 and $37 \mathrm{~km} \mathrm{~s}^{-1}$ (blueshifted component). The contour levels are $5,10,15$ and $20 \mathrm{~K} \mathrm{~km} \mathrm{~s}^{-1}$. The thick contours show the ${ }^{12} \mathrm{CO} J=3-2$ emission integrated between 50 to $63 \mathrm{~km} \mathrm{~s}^{-1}$ (redshifted component) and the levels are $10,15,20$ and $25 \mathrm{~K} \mathrm{~km} \mathrm{~s}^{-1}$. The crosses indicate the positions of the 2MASS sources IR1 and IR2.

Figure 12 (left) displays the ${ }^{12} \mathrm{CO}$ position-velocity diagram along a line at constant Right Ascension $\left(18^{\mathrm{h}} 56^{\mathrm{m}} 48^{\mathrm{s}}\right)$, while Fig. 12 (right) shows the same diagram along a line at constant Declination $\left(+01^{\circ} 18^{\prime} 45^{\prime \prime}\right)$. Redshifted and blueshifted features extending in velocity are clearly evident in both images.

Based on these observational results we can confirm that we are observing gas outflows originating in the source IR1. These results together with the above noticed slightly extended emission in the IRAC $4.5 \mu \mathrm{m}$ band (indicative of gas shocked by outflows) and its position in the infrared color-color diagram (see Sect. 3.1), confirm that IR1 is a YSO. In Fig. 13 we present a three dimensional picture of the $\mathrm{HCO}^{+}$structure and the 

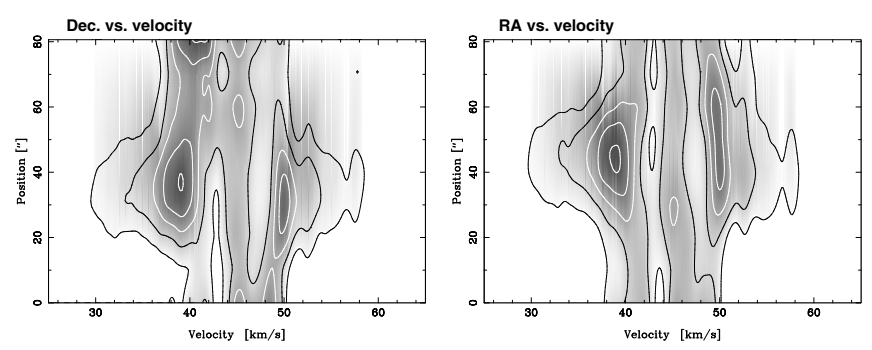

Fig. 12. ${ }^{12} \mathrm{CO}$ position-velocity diagrams. Left: along a line at constant $\mathrm{RA}=18^{\mathrm{h}} 56^{\mathrm{m}} 48^{\mathrm{s}}$. Right: along a line at constant dec. $=+01^{\circ} 18^{\prime} 45^{\prime \prime}$.

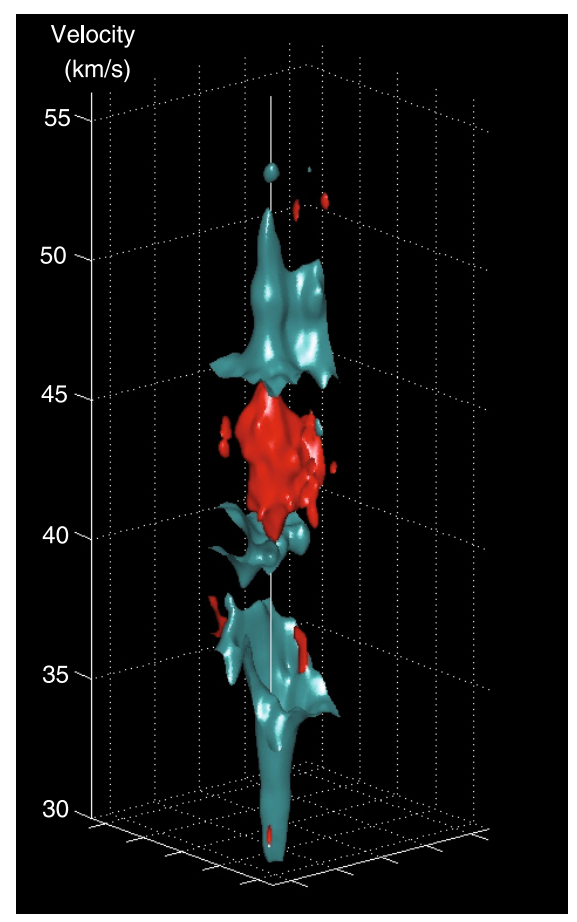

Fig. 13. Three dimensional picture showing isosurfaces of intensity. The feature between $\sim 40$ and $45 \mathrm{~km} \mathrm{~s}^{-1}$ is the $\mathrm{HCO}^{+}$emission at $0.7 \mathrm{~K}$ while the extended features in velocity (along the vertical axis) show the isosurface of the ${ }^{12} \mathrm{CO}$ emission at $1.8 \mathrm{~K}$.

outflows mapped by the ${ }^{12} \mathrm{CO}$. The figure shows the ${ }^{12} \mathrm{CO}$ and the $\mathrm{HCO}^{+}$data as isosurfaces of intensity. The vertical axis corresponds to the velocity while the horizontal axes represent the spatial coordinates. The feature at $v \sim 40-45 \mathrm{~km} \mathrm{~s}^{-1}$ is the $\mathrm{HCO}^{+}$emission at $0.7 \mathrm{~K}$. The extended features in velocity are the ${ }^{12} \mathrm{CO}$ emission at $1.8 \mathrm{~K}$, which clearly show the outflow structure.

\subsection{3. ${ }^{13} \mathrm{CO} J=3-2$ and $\mathrm{CS} J=7-6$}

Figure 14 displays the profiles of the ${ }^{13} \mathrm{CO} J=3-2$ and CS $J=7-6$ emission, left and right, respectively, obtained towards the center of the studied region $\left(R A=18^{\mathrm{h}} 56^{\mathrm{m}} 47.8^{\mathrm{s}}\right.$, dec. $\left.=+01^{\circ} 18^{\prime} 45^{\prime \prime}, \mathrm{J} 2000\right)$. The parameters determined from Gaussian fitting of these lines are presented in Table 2. $T_{\mathrm{mb}}$ represents the peak brightness temperature, $V_{\mathrm{lsr}}$ the central velocity, $\Delta v$ the line width and $I$ the integrated line intensity. Errors are formal $1 \sigma$ values for the model of a Gaussian line shape.

We have calculated the ratio of the integrated line intensities between the ${ }^{13} \mathrm{CO} J=3-2$ and $J=1-0$ lines $\left({ }^{13} R_{3-2 / 1-0}\right)$. The latter was extracted from the GRS (Jackson et al. 2006) and the
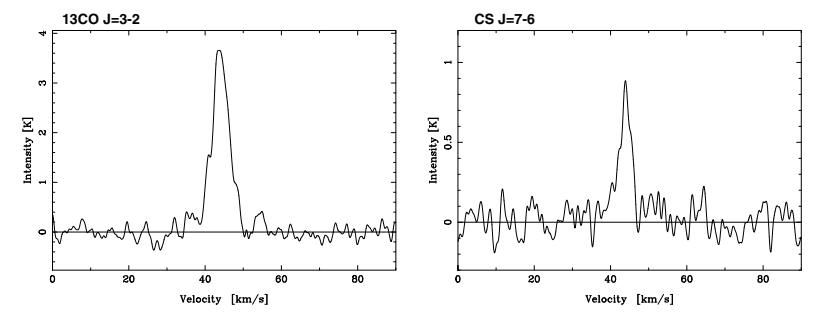

Fig. 14. ${ }^{13} \mathrm{CO} J=3-2$ profile (left) and CS $J=7-6$ profile (right) obtained towards RA $=18^{\mathrm{h}} 56^{\mathrm{m}} 47.8^{\mathrm{s}}$, dec. $=+01^{\circ} 18^{\prime} 45^{\prime \prime}(\mathrm{J} 2000)$. The rms noise is $\sigma_{\text {rms }} \sim 0.10$ and $0.07 \mathrm{~K}$ for each line, respectively.

Table 2. Observed parameters of the ${ }^{13} \mathrm{CO} J=3-2$ and CS $J=7-6$ emissions towards RA $=18^{\mathrm{h}} 56^{\mathrm{m}} 47.8^{\mathrm{s}}$, dec. $=+01^{\circ} 18^{\prime} 45^{\prime \prime}(\mathrm{J} 2000)$.

\begin{tabular}{ccccc}
\hline \hline Emission & $\begin{array}{c}T_{\mathrm{mb}} \\
(\mathrm{K})\end{array}$ & $\begin{array}{c}V_{\mathrm{lsr}} \\
\left(\mathrm{km} \mathrm{s}^{-1}\right)\end{array}$ & $\begin{array}{c}\Delta v \\
\left(\mathrm{~km} \mathrm{~s}^{-1}\right)\end{array}$ & $\begin{array}{c}I \\
\left(\mathrm{~K} \mathrm{~km} \mathrm{~s}^{-1}\right)\end{array}$ \\
\hline${ }^{13} \mathrm{CO} J=3-2$ & $3.55 \pm 0.30$ & $44.10 \pm 0.20$ & $5.45 \pm 0.50$ & $21 \pm 2$ \\
$\mathrm{CS} J=7-6$ & $0.50 \pm 0.10$ & $43.80 \pm 0.90$ & $4.00 \pm 0.40$ & $2.2 \pm 0.5$ \\
\hline
\end{tabular}

$J=3-2$ line was convolved to the $J=1-0$ beam. We obtain ${ }^{13} R_{3-2 / 1-0} \sim 1.4$.

In order to compare the ${ }^{13} \mathrm{CO} J=3-2$ with the ${ }^{12} \mathrm{CO} J=3-2$ to obtain an isotopic ratio between peak temperatures for the different velocity components, we fit the ${ }^{13} \mathrm{CO} J=3-2$ spectrum with three Gaussians. We use the Gaussians with lower and higher velocities, closer to the blueshifted and redshifted components, respectively, as seen in the ${ }^{12} \mathrm{CO}$ emission (see Fig. 10). Thus we obtain the following peak temperature ratios: ${ }^{12} T /{ }^{13} T \sim 13.5$ and ${ }^{12} T /{ }^{13} T \sim 8.8$ for the emissions close to the blueshifted and redshifted components, respectively. From these ratios we derive the optical depths for each line. For this region of the Galaxy, according to Milam et al. (2005), we use an isotopic abundance ratio of $\left[{ }^{12} \mathrm{CO}\right] /\left[{ }^{13} \mathrm{CO}\right] \sim 70$. Assuming an excitation temperature of $T_{\mathrm{ex}} \sim 20 \mathrm{~K}$ for both lines, we derive the following optical depths: $\tau^{12} \sim 6$ and $\tau^{13} \sim 0.07$ for the emission close to the blueshifted component and $\tau^{12} \sim 10$ and $\tau^{13} \sim 0.1$ for the emission close to the redshifted one.

\subsection{Kinematics and dynamics of the outflows}

In this section we investigate the physical parameters of the discovered outflows. To carry out these calculations we applied the relations from Choi et al. (1993). The CO column density $\left(N_{i}^{\mathrm{CO}}\right)$ and the mass $\left(M_{i}\right)$ for each channel are calculated from:

$N_{i}^{\mathrm{CO}}=1.1 \times 10^{15} \frac{T_{3-2} \Delta v}{D\left(n, T_{k}\right)} \frac{\tau_{32}}{1-\exp \left(-\tau_{32}\right)}$ and

$M_{i}=\mu \mathrm{m}_{H_{2}} \mathrm{~d}^{2} \Omega N_{i}^{\mathrm{CO}} \frac{[\mathrm{H}]}{[\mathrm{C}]} \frac{[\mathrm{C}]}{[\mathrm{CO}]}$,

where

$D\left(n, T_{k}\right)=f_{2}\left[J_{v}\left(T_{\mathrm{ex}}\right)-J_{v}\left(T_{\mathrm{bk}}\right)\right]\left[1-\exp \left(-16.597 / T_{\mathrm{ex}}\right)\right]$,

$T_{3-2}$ is the peak temperature of the ${ }^{12} \mathrm{CO} J=3-2$ line, $\Delta v$ the channel width, $f_{2}$ is the fraction of $\mathrm{CO}$ molecules in the $J=$ 2 state, $d=3 \mathrm{kpc}$ is the distance to the giant molecular cloud, $\Omega$ is the solid angle subtended by emission, $[\mathrm{H}] /[\mathrm{C}]=2.5 \times$ $10^{3}$ (Grevesse et al. 1991), [C]/[CO] = 8 (Dickman 1978; van Dishoeck et al. 1992), and $\tau_{32}$ is the optical depth of the ${ }^{12} \mathrm{CO}$ $J=3-2$ transition calculated above, a value of 1.5 is adopted 
Table 3. Outflows parameters.

\begin{tabular}{lccccccc}
\hline \hline Shift & $\begin{array}{c}R \\
(\mathrm{pc})\end{array}$ & $\begin{array}{c}M \\
\left(M_{\odot}\right)\end{array}$ & $\begin{array}{c}t_{\text {dyn }} \\
(\mathrm{yr})\end{array}$ & $\begin{array}{c}\dot{M} \\
\left(M_{\odot} \mathrm{yr}^{-1}\right)\end{array}$ & $\begin{array}{c}P \\
\left(M_{\odot} \mathrm{km} \mathrm{s}^{-1}\right)\end{array}$ & $\begin{array}{c}E_{k} \\
\left(M_{\odot}\left[\mathrm{km} \mathrm{s}^{-1}\right]^{2}\right)\end{array}$ & $\begin{array}{c}F \\
\left(M_{\odot} \mathrm{km} \mathrm{s}^{-1} \mathrm{yr}^{-1}\right)\end{array}$ \\
\hline Blue & 0.35 & $7.0 \times 10^{-4}$ & $2.6 \times 10^{4}$ & $2.7 \times 10^{-8}$ & $7.7 \times 10^{-3}$ & $4.4 \times 10^{-2}$ & $3.0 \times 10^{-7}$ \\
Red & 0.30 & $1.4 \times 10^{-4}$ & $4.2 \times 10^{4}$ & $3.3 \times 10^{-8}$ & $1.2 \times 10^{-2}$ & $5.5 \times 10^{-2}$ & $2.7 \times 10^{-7}$ \\
\hline
\end{tabular}

for $D\left(n, T_{k}\right)$ following Choi et al. (1993). With these parameters we have estimated the mass $M$, the momentum $P$ and the kinetic energy $E_{k}$ for each outflow from:

$$
\begin{aligned}
& M=\sum_{i} M_{i}, \\
& P=\sum_{i} M_{i}\left|v_{i}-v_{0}\right|, \\
& E_{k}=\sum_{i} \frac{1}{2} M_{i}\left(v_{i}-v_{0}\right)^{2},
\end{aligned}
$$

where $v_{0}$ is the systemic velocity, $\sim 45 \mathrm{~km} \mathrm{~s}^{-1}$. Finally we calculate the dynamical timescale $t_{\mathrm{dyn}}=R / V_{\mathrm{ch}}$, where $V_{\mathrm{ch}}=P / M$ is the characteristic velocity and $R$ is the size of the outflows, the mass loss rate $\dot{M}=M / t_{\text {dyn }}$ and the average driving force $F=P / t_{\text {dyn }}$. As the outflows are seen along the line of sight, that is the blue lobe is superposed on the red lobe, we estimate their sizes $R$ from the FWHM contour (see Fig. 11). The results are presented in Table 3.

\section{Discussion}

From the 2MASS data study we found that the sources with the highest NIR excess lie on the border of the HII region G034.8-0.7 and around the dark cloud IRDC 34.776-0.554, a scenario that strongly suggests active star formation. The source 2MASS $18564827+0118471$ (IR1 in this paper) is the most reddened source in the NIR and is also bright and slightly extended in the IRAC $4.5 \mu \mathrm{m}$ band. This latter emission, which contains $\mathrm{H}_{2}$ and $\mathrm{CO}$ vibrational lines, can be produced when protostellar outflows crash into the ambient ISM, suggesting that IR1 could be a YSO.

Our molecular observations have revealed the existence of a $\mathrm{HCO}^{+}$clump where IR1 is embedded. It is known that such molecular species is enhanced in molecular outflows (Rawlings et al. 2004). In effect, a strong enhancement of the $\mathrm{HCO}^{+}$abundance is expected to occur in the boundary layer between the outflow jet and the surrounding molecular core. This would be due to the liberation and photoprocessing by the shock of the molecular material stored in the icy mantles of the dust. Indeed, through the ${ }^{12} \mathrm{CO} J=3-2$ line we discovered the presence of outflows extended along the line of sight related to the $\mathrm{HCO}^{+}$clump and IR1. In this context, we conclude that the source IR1 is a YSO driving bipolar outflows which are enhancing the abundance of $\mathrm{HCO}^{+}$.

The obtained outflow masses are similar to those obtained for the outflows of the source IRS6, a low-mass YSO in Cederblad 110 (Hiramatsu et al. 2007). We compared the derived parameters for the outflows (see Table 3) with those of the statistical study carried out by Wu et al. (2004) based on the properties of a sample of about 391 molecular outflows. In that work the sources were divided into outflows with low mass and outflows with high mass according to either the available bolometric luminosity of the central source or the outflow mass.
Based on the compiled data the authors conclude that the outflow phenomenon is common either in low and high mass star formation regions. The red and blue outflow masses that we derived for IR1 are slightly lower than the lowest masses discussed by Wu et al. (2004). Therefore, IR1 would correspond to the "low mass group" as catalogued by them. It is important to remark that since we made no corrections to the velocities for possible projection effects, the momenta and kinetic energies that we derive are lower limits. Also, as Choi et al. (1993) note, these effects can increase (if the flow is in the plane of the sky) or decrease (if the flow is along the line of sight) the timescale ( $t_{\text {dyn }}$ in Table 3 ) by substantial factors. In addition, this time would be shorter if we use the maximum velocity rather than $V_{\mathrm{ch}}$. However our $t_{\text {dyn }}$ values are very close to the average dynamical time of the "low mass group" presented in Wu et al. (2004). Other physical parameters, like kinetic energy, momentum and driving force listed in Table 3 also agree with those of the "low mass group". We can therefore conclude that we are probably observing a source with outflows of low mass.

One stage in the formation of a star is the main accretion phase. This is the phase during which the central object builds up its mass from a surrounding infalling envelope and accretion disk. Observational evidence shows that the main accretion phase is always accompanied by a powerful ejection of a small fraction of the accreted material in the form of prominent bipolar outflows (e.g. McKee \& Ostriker 2007; Bachiller 1996). Class 0 protostars are considered the earlier stage of star formation with typical ages of $1-3 \times 10^{4}$ years. Most of them, if not all, drive powerful "jet-like" CO molecular outflows, which gradually disappear as the protostar goes into the following evolutionary stages. Thus, the dynamical times of the outflows can be used as an approximation of the life time of YSOs. Therefore we suggest an age of a few $\times 10^{4}$ years for IR1.

Additionally, we performed a Spectral Energy Distribution (SED) fit of IR1 near and mid IR fluxes extracted from the 2MASS Point Source Catalog ( $J, H$ and $K$ bands), from the Glimpse Catalog (Spitzer-IRAC bands) and from the MSX Point Source Catalog (8.28, 12.13, 14.65 and $21.30 \mu \mathrm{m}$ bands). Based on the SED models ${ }^{2}$ presented by Robitaille et al. (2006, 2007) and considering a visual extinction between 15 and 30 mag (see Sect. 3.1), we suggest that IR1 can be a high mass YSO of $\sim 20 M_{\odot}$. Besides, the models predict an age between $7 \times 10^{4}$ and $10^{5}$ years for this YSO, and a circumstellar disk of $0.01 M_{\odot}$ with an accretion rate of $\sim 10^{-6} M_{\odot} \mathrm{yr}^{-1}$. The outflows and accretion rates presented in Table 3 and derived from the SED models, respectively, are considerably lower than the typical rates of massive YSOs $\left(10^{-4} M_{\odot} \mathrm{yr}^{-1}\right.$ in both cases; McKee \& Tan 2003). As noticed above, IR1 presents a high NIR excess, which suggests that it is embedded in a very dense envelope. Taking into account the YSO mass obtained from the SED models, the estimated ages and the mentioned outflow and accretion rates for IR1, we suggest that this source is probably a massive YSO that could be near the end of the accretion process and has not ionized and dissipated its envelope yet.

$\overline{2}$ http://caravan.astro.wisc.edu/protostars 
The integrated line ${ }^{13} \mathrm{CO} 3-2 / 1-0$ ratio $\left({ }^{13} R_{3-2 / 1-0}\right)$ obtained towards the center of the region is very similar to that obtained towards a circumstellar disk around a pre-main sequence star by van Zadelhoff et al. (2001). As the authors remark, the ${ }^{13} R_{3-2 / 1-0}$ could indicate temperatures of $\sim 20-40 \mathrm{~K}$. However, care should be taken with the interpretation of this result since the emission of the two lines most likely comes from different regions of the disk due to the difference in optical depth of the two lines.

The sulphur-bearing species, like the CS, have been associated with shocks and/or outflows. However it is still debatable whether sulphur-bearing species are good diagnostics of outflows (Johnstone et al. 2003). According to Moriarty-Schieven et al. (1995), the CS $J=7-6$ line traces high densities $\left(>10^{7} \mathrm{~cm}^{-3}\right)$ and warm temperatures $(>60 \mathrm{~K})$ in the protostellar envelopes. Thus, we propose that the detection of this transition indicates the presence of dense and warm gas, showing the inner denser and warmer part of the protostellar envelope where IR1 is forming.

\subsection{Could the SNR W44 or the HII region G034.8-0.7 have triggered the formation of IR1?}

From our molecular results and the IR emission study, we conclude that IR1 is indeed a YSO. As it lies on the border of the HII region G034.8-0.7, which is in turn evolving in a molecular cloud shocked by the SNR W44, we investigate their relatedness.

According to the derived outflow dynamical times (see Table 3) and to the SED age estimate, IR1 might be a YSO with an age of a few $\times 10^{4}$ or maybe $\sim 10^{5}$ years. Based on the age of the associated pulsar, Wolszczan et al. (1991) estimated an age of about $2 \times 10^{4}$ years for the SNR W44. Taking into account that the ages of IR1 and W44 are comparable and that a delay between the main triggering agent and the subsequent star formation is expected, it is unlikely that the SNR triggered the formation of IR1.

On the other hand, as mentioned above, IR1 is seen in the plane of the sky overlapping the photodissociation region (PDR) associated with the HII region G034.8-0.7, located at the same distance as W44 (Ortega et al. 2007). In the literature there are many works about star formation triggered by the expansion of HII regions (e.g. Elmegreen \& Lada 1977; Deharveng et al. 2003, 2005; Comerón et al. 2005; Zavagno et al. 2006). In what follows we investigate this possibility. From the ${ }^{12} \mathrm{CO} J=1-0$ study performed by Seta et al. (2004), a dense molecular shell surrounding the HII region G034.8-0.7 is evident in the velocity range $35-40 \mathrm{~km} \mathrm{~s}^{-1}$, which suggests that in this region the "collect and collapse" process can take place (Deharveng et al. 2005; Zavagno et al. 2006). In this process, the compressed shocked layer generated in the inhomogeneous medium by the expansion of the HII region may become gravitationally unstable along its surface on a long timescale. The process produces massive fragments of material which allow the formation of massive stars and/or clusters. According to Ortega et al. (2007), taking into account that the exciting star of the HII region G034.8-0.7 should be of spectral type between $\mathrm{O} 9.5$ and O3, the age of the HII region would be $10^{5}-3 \times 10^{6}$ years. These times are compatible with star forming processes in the associated PDR. Besides, as noticed in Sect. 3.1, the most reddened sources in the analyzed region lie preferentially on the border of the HII region that is seen in projection interior to the W44 shell. We can therefore conclude that a scenario where the expansion of the HII region G034.8-0.7 has triggered the formation of IR1 is more possible. Careful modeling of this scenario, including the actions of the HII region and the SNR, is planned for the near future.
Future molecular observations are planned to be performed towards this region in order to look for more YSOs embedded in the perturbed molecular cloud. Also extended CS observations towards IR1 would be important. The transitions of this molecular species are very useful to derive the detailed density structure within the molecular envelopes of star-forming regions.

\section{Summary}

We present an infrared study and molecular observations towards the IR source IRAS $18542+0114$ carried out to explore signatures of star forming activity on the border of the HII region G034.8-0.7, which evolves within a molecular cloud shocked by the SNR W44. We found that IRAS $18542+0114$ presents a slight extended emission in the IRAC $4.5 \mu \mathrm{m}$ band, which suggests that this source may be a YSO driven outflow. IRAS $18542+0114$ is resolved into several sources in the 2MASS All-Sky Point Source Catalog, and two of them according to color criteria are YSO candidates. One of these candidates, 2MASS $18564827+0118471$ (IR1 in this work), is the most reddened source in the NIR in the studied region. From SED models we suggest that IR1 is probably a massive YSO that could be near the end of the accretion process. Our molecular results confirm that IR1 is indeed a YSO. These results can be summarized as follows:

(a) We discovered a $\mathrm{HCO}^{+}$clump towards IR1. It is known that such molecular species is enhanced in molecular outflows, which is strong evidence of star forming activity.

(b) We discovered ${ }^{12} \mathrm{CO}$ outflows towards IR1 extended along the line of sight. From the dynamical time of these outflows and an SED age estimate, we suggest an age of a few $\times 10^{4}$ or maybe $\sim 10^{5}$ years for IR1. The discovery of molecular outflows confirms that IR1 is a YSO.

(c) From the detection of the CS $J=7-6$ line we confirm the presence of high density $\left(>10^{7} \mathrm{~cm}^{-3}\right)$ and warm $(>60 \mathrm{~K})$ gas towards IR1, probably belonging to the protostellar envelope where this YSO is forming.

(d) By comparing the estimated age for IR1 and the age of the SNR W44 ( $2 \times 10^{4}$ years), we conclude that it is unlikely that the SNR triggered the formation of IR1. On the other hand, taking into account the age of the HII region G034.8-0.7 and that IR1, as other reddened sources, lie on its border, we find that the HII region may have initiated star formation through the "collect and collapse" process.

Future observations and theoretical modeling are planned in order to further investigate the star formation in this complex region.

Acknowledgements. We wish to thank the referee Dr. Zavagno whose constructive criticism has helped to improve the paper. S.P. is grateful to the staff of ASTE for the support received during the observations, especially to Juan Cortés. S.P. acknowledges the support of Viviana Guzmán during the observations. S.P. is a postdoctoral fellow of CONICET, Argentina. M.O. is a doctoral fellow of CONICET, Argentina. G.D. is member of the Carrera del Investigador Cientifico of CONICET, Argentina. This work was partially supported by the CONICET grant 6433/05, UBACYT A023 and ANPCYT PICT 04-14018. M.R. is supported by the Chilean Center for Astrophysics FONDAP No. 15010003. M.R. and S.P. aknowledge support from FONDECYT N ${ }^{\circ} 1080335$.

\section{References}

Allen, L. E., Calvet, N., D’Alessio, P., et al. 2004, ApJS, 154, 363 Bachiller, R. 1996, ARA\&A, 34, 111

Bessell, M. S., \& Brett, J. M. 1988, PASP, 100, 1134 
Caswell, J. L., Murray, J. D., Roger, R. S., Cole, D. J., \& Cooke, D. J. 1975, A\&A, 45, 239

Choi, M., Evans, II, N. J., \& Jaffe, D. T. 1993, ApJ, 417, 624

Comerón, F., Schneider, N., \& Russeil, D. 2005, A\&A, 433, 955

Cutri, R. M., Skrutskie, M. F., van Dyk, S., et al. 2003, 2MASS All Sky Catalog of point sources. (The IRSA 2MASS All-Sky Point Source Catalog, NASA/IPAC Infrared Science Archive, http://irsa.ipac.caltech. edu/applications/Gator/)

Cyganowski, C. J., Whitney, B. A., Holden, E., et al. 2008, AJ, 136, 2391

Deharveng, L., Zavagno, A., Salas, L., et al. 2003, A\&A, 399, 1135

Deharveng, L., Zavagno, A., \& Caplan, J. 2005, A\&A, 433, 565

Dickman, R. L. 1978, ApJS, 37, 407

Elmegreen, B. G., \& Lada, C. J. 1977, ApJ, 214, 725

Ezawa, H., Kawabe, R., Kohno, K., \& Yamamoto, S. 2004, SPIE Conf. Ser., 5489, ed. J. M. Oschmann, Jr., 763

Fazio, G. G., Hora, J. L., Allen, L. E., et al. 2004, ApJS, 154, 10

Gregersen, E. M., Evans, II, N. J., Zhou, S., \& Choi, M. 1997, ApJ, 484, 256

Gregersen, E. M., Evans, II, N. J., Mardones, D., \& Myers, P. C. 2000, ApJ, 533, 440

Grevesse, N., Lambert, D. L., Sauval, A. J., et al. 1991, A\&A, 242, 488

Hanson, M. M., Howarth, I. D., \& Conti, P. S. 1997, ApJ, 489, 698

Helfand, D. J., Becker, R. H., White, R. L., Fallon, A., \& Tuttle, S. 2006, AJ, 131,2525

Hiramatsu, M., Hayakawa, T., Tatematsu, K., et al. 2007, ApJ, 664, 964

Hoffman, I. M., Goss, W. M., Brogan, C. L., \& Claussen, M. J. 2005, ApJ, 627, 803

Jackson, J. M., Rathborne, J. M., Shah, R. Y., et al. 2006, ApJS, 163, 145

Johnstone, D., Boonman, A. M. S., \& van Dishoeck, E. F. 2003, A\&A, 412, 157

Kuchar, T. A., \& Clark, F. O. 1997, ApJ, 488, 224

Leung, C. M., \& Brown, R. L. 1977, ApJ, 214, L73

Lockman, F. J. 1989, ApJS, 71, 469

Mac Low, M.-M., \& Klessen, R. S. 2004, Rev. Mod. Phys., 76, 125

McKee, C. F., \& Ostriker, E. C. 2007, ARA\&A, 45, 565

McKee, C. F., \& Tan, J. C. 2003, ApJ, 585, 850

Melioli, C., de Gouveia Dal Pino, E. M., de La Reza, R., \& Raga, A. 2006, MNRAS, 373, 811
Milam, S. N., Savage, C., Brewster, M. A., Ziurys, L. M., \& Wyckoff, S. 2005, ApJ, 634, 1126

Moriarty-Schieven, G. H., Wannier, P. G., Mangum, J. G., Tamura, M., \& Olmsted, V. K. 1995, ApJ, 455, 190

Opik, E. J. 1953, Irish Astron. J., 2, 219

Ortega, M. E., Cichowolski, S., Castelletti, G., \& Dubner, G. 2007, Boletín de la Asociación Argentina de Astronomía, La Plata, Argentina, 50, 189

Radhakrishnan, V., Brooks, J. W., Goss, W. M., Murray, J. D., \& Schwarz, U. J. 1972, ApJS, 24, 1

Rathborne, J. M., Jackson, J. M., \& Simon, R. 2006, ApJ, 641, 389

Rathborne, J. M., Simon, R., \& Jackson, J. M. 2007, ApJ, 662, 1082

Rawlings, J. M. C., Redman, M. P., Keto, E., \& Williams, D. A. 2004, MNRAS, 351,1054

Reach, W. T., Rho, J., Tappe, A., et al. 2006, AJ, 131, 1479

Rieke, G. H., \& Lebofsky, M. J. 1985, ApJ, 288, 618

Robitaille, T. P., Whitney, B. A., Indebetouw, R., Wood, K., \& Denzmore, P. 2006, ApJS, 167, 256

Robitaille, T. P., Whitney, B. A., Indebetouw, R., \& Wood, K. 2007, ApJS, 169, 328

Seta, M., Hasegawa, T., Dame, T. M., et al. 1998, ApJ, 505, 286

Seta, M., Hasegawa, T., Sakamoto, S., et al. 2004, AJ, 127, 1098

Skrutskie, M. F., Cutri, R. M., Stiening, R., et al. 2006, AJ, 131, 1163

van Dishoeck, E. F., \& Jørgensen, J. K. 2008, Ap\&SS, 313, 15

van Dishoeck, E. F., Glassgold, A. E., Guelin, M., et al. 1992, in Astrochemistry of Cosmic Phenomena, ed. P. D. Singh, IAU Symp., 150, 285

van Zadelhoff, G.-J., van Dishoeck, E. F., Thi, W.-F., \& Blake, G. A. 2001, A\&A, 377,566

Vanhala, H. A. T., \& Cameron, A. G. W. 1998, ApJ, 508, 291

Werner, M. W., Roellig, T. L., Low, F. J., et al. 2004, ApJS, 154, 1

Wolszczan, A., Cordes, J. M., \& Dewey, R. J. 1991, ApJ, 372, L99

Wu, Y., Wei, Y., Zhao, M., et al. 2004, A\&A, 426, 503

Zavagno, A., Deharveng, L., Comerón, F., et al. 2006, A\&A, 446, 171

Zhou, S. 1992, ApJ, 394, 204

Zhou, S., Evans, II, N. J., Koempe, C., \& Walmsley, C. M. 1993, ApJ, 404, 232 